\title{
Towards an Interactive Experiment Framework: DynamiQ
}

\author{
Razvan Beuran \\ Japan Advanced Institute of \\ Science and Technology \\ Japan \\ razvan@jaist.ac.jp \\ Yuuki Takano \\ National Institute of \\ Information and \\ Communications Technology \\ Japan \\ ytakano@nict.go.jp
}

\author{
Shingo Yasuda \\ National Institute of \\ Information and \\ Communications Technology \\ Japan \\ s-yasuda@nict.go.jp \\ Toshiyuki Miyachi \\ National Institute of \\ Information and \\ Communications Technology \\ Japan \\ miyachi@nict.go.jp
}

\author{
Tomoya Inoue \\ Japan Advanced Institute of \\ Science and Technology \\ Japan \\ t-inoue@jaist.ac.jp \\ Yoichi Shinoda \\ Japan Advanced Institute of \\ Science and Technology \\ Japan \\ shinoda@jaist.ac.jp
}

\begin{abstract}
Interactive network experiments are useful for finding solutions to network problems, for teaching and for training purposes. In this demonstration we shall present an interactive experiment framework that allows users to directly control the experiment scenario by using a touch panel interface. This framework uses the network emulation module named dynamiQ for the dynamic emulation of networks.

The demonstration uses a scenario with up to $55 \mathrm{emu}-$ lated nodes, out of which 30 nodes form an emulated vehicular network and 5 are static buildings. The other nodes represent wireless towers and unmanned aerial vehicles that can be freely placed in the virtual experiment area. Participants are tasked with creating a multi-hop mesh network for sending video traffic between two predefined remote locations. A similar demonstration has already been shown at Interop Tokyo 2014, where it received a special jury award.
\end{abstract}

\section{Categories and Subject Descriptors}

C.4 [Performance of Systems]: Measurement techniques

\section{General Terms}

Experimentation, Performance, Verification

\section{Keywords}

Interactive experiments, dynamic experiment scenarios, network emulation

* Razvan Beuran is also with the National Institute of Information and Communications Technology (NICT), Japan. This work was done when he was mainly with NICT.

Permission to make digital or hard copies of all or part of this work for personal or classroom use is granted without fee provided that copies are not made or distributed for profit or commercial advantage and that copies bear this notice and the full citation on the first page. To copy otherwise, to republish, to post on servers or to redistribute to lists, requires prior specific permission and/or a fee.

TRIDENTCOM'15 Vancouver, Canada

Copyright 2015 ACM X-XXXXX-XX-X/XX/XX ...\$15.00.

\section{INTRODUCTION}

Network experiments are usually performed based on predefined scenarios, especially in the case of simulation and emulation. This is the most straightforward approach, but it requires deciding in advance all the experiment conditions: number and position of the nodes, including their mobility, properties of the communication environment, traffic, etc.

Interactive experiments are most appropriate when searching for the solution to a certain practical problem, such as the wireless network planning for a certain geographic area. Network experts could use an interactive experiment platform to virtually deploy various network solutions and analyze their cost and performance characteristics, without the time overhead, risks and expenses related to the actual deployment of alternative solutions.

Interactive experiments can also be used for training purposes, as a means to provide an affordable yet valuable hands-on experience with network resources that are otherwise difficult to manipulate in practice.

In this paper we present a demonstration that uses the interactive experiment framework that we designed and implemented for a practical mesh network planning problem: how to construct a multi-hop network topology so that video can be successfully delivered between two end nodes. A video camera and a display are located on the demonstration premises, whereas the emulated network is run on a set of high-performance servers. Participants use a touch screen interface to interact with the experiment framework and create the required mesh network.

\section{FRAMEWORK OVERVIEW}

In this section we outline the design of the proposed interactive experiment framework. For a detailed description see the companion paper at TridentCom 2015 entitled "DynamiQ: A Tool for Dynamic Emulation of Networks".

\subsection{Infrastructure}

An interactive experiment framework such as the one we propose requires an infrastructure with sufficient capabilities to meet all the execution requirements. We use the network emulation approach, since it makes it possible to employ real 


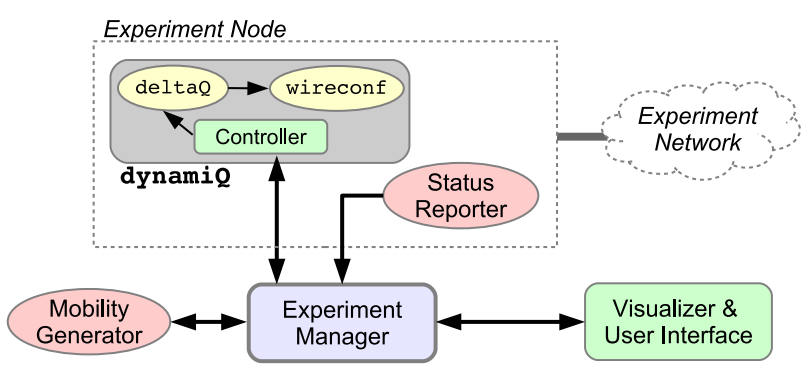

Figure 1: Architecture of the interactive experiment framework.

applications and protocols in the experiments. Moreover, emulation tools are designed to run in real time, a necessary feature when dealing interactively with user input.

\subsubsection{StarBED}

StarBED is a network testbed located at the Hokuriku StarBED Technology Center of the National Institute of Information and Communications Technology, Japan [5].

StarBED makes available for experiments more than 1400 interconnected PCs, and represents the physical infrastructure of our interactive experiment framework. The control and the experiment networks of StarBED are independent of each other, so as to prevent traffic interference.

\subsubsection{QOMET}

QOMET is a set of tools for network emulation targeting mainly wireless networks [1]. It uses an XML-based user-defined scenario as input, which describes the network environment, including node properties and mobility, etc. QOMET provides the necessary mechanisms for performing experiments in a distributed manner by reproducing on StarBED the communication conditions between the wireless nodes in the scenario [2].

Emulation using QOMET is performed as a two-stage process. First of all, the module called deltaQ will process the input scenario and compute the communication conditions between nodes. Then, the module named wireconf will recreate the communication conditions between scenario nodes in the StarBED experiment network. For this we use of the link emulation system ipfw, which can artificially introduce packet loss, delay and bandwidth limitations [3].

\subsection{Framework architecture}

Figure 1 presents the architecture of the interactive experiment framework, its components and their interactions.

\subsubsection{Experiment Manager}

The experiments in our framework are managed by a central module named Experiment Manager. Its role is to multiplex the information from different sources, and to drive the execution of the framework components.

\subsubsection{DynamiQ}

In order to support dynamic emulation of networks it is necessary to have a network emulation component that can handle scenario reconfiguration in real time, so that both the internal scenario changes and the network communication condition changes are applied in a timely fashion; thus

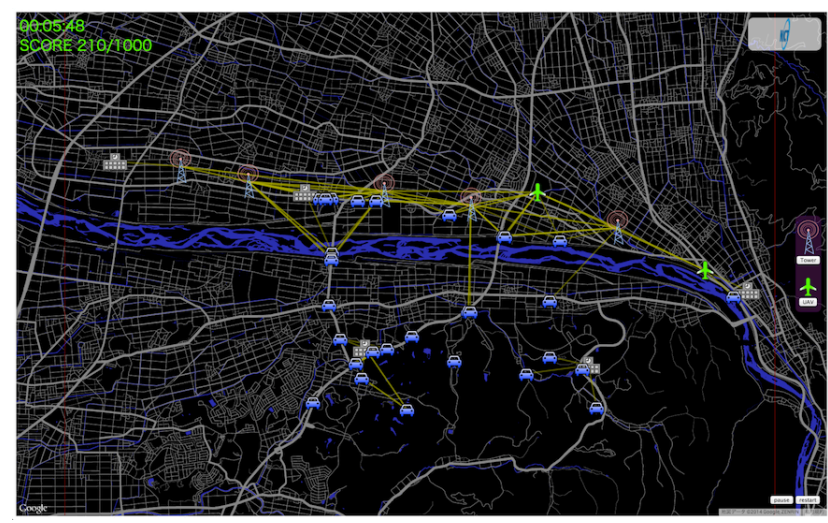

Figure 2: The 2D visualizer and user interface.

the user can get continuous feedback from the experiment framework.

The dynamic network emulation functionality is implemented by the module called dynamiQ (dynamic QOMET). DynamiQ effectively provides the network emulation functionality of QOMET but in a dynamic manner, without a predefined scenario as its static counterpart. This is done by leveraging the functionality of the two main QOMET modules, deltaQ and wireconf, and by adding a Controller module to manage them.

\subsubsection{Visualizer \& User Interface}

The Visualizer $\&$ User Interface module handles the display of the experiment state to the user, but it also gets user input for controlling the experiment flow. Thus, one can add nodes to the experiment, modify their position or settings, then see how these changes affect the experiment, for instance, how the communication conditions vary.

In order to effectively deal with user input, experiment status must be visualized continuously and in real time. In our current implementation, a $2 \mathrm{D}$ visualizer is used to provide a top view of the experiment area. The $2 \mathrm{D}$ visualizer is coupled with the user interface to provide input to the interactive framework (see Figure 2).

Since our modular architecture allows using multiple visualizers simultaneously, we have also implemented a 3D visualizer (see Figure 3). The 3D visualizer provides a side view of the experiment area, thus offering a deeper insight into the experiment conditions. This is useful especially when area topography and node height are important for the experiment scenario.

\subsubsection{Status Reporter}

The role of the Status Reporter is to gather status information from the live experiment network, which is then used by the other framework components. At this time such information is mainly employed for visualization purposes.

Currently the Status Reporter functionality focuses on collecting information on the topology of the mesh network that is constructed in our demonstration by using the OLSR routing daemon named olsrd [6].

\subsubsection{Mobility Generator}

In the current implementation we employ the ONE simulator [4] as a mobility generator. In particular, we use 


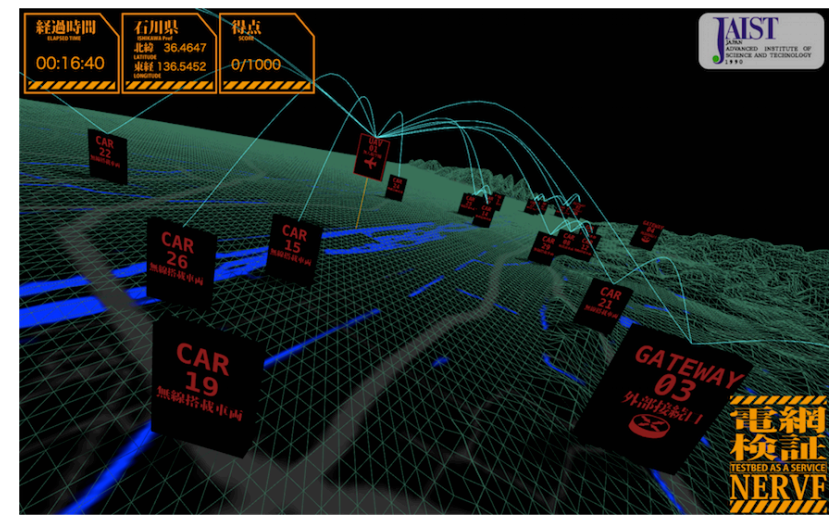

Figure 3: The 3D visualizer.

the Shortest Path Map Based Movement (SPMBM) model of ONE to generate trajectories of mobile nodes given the road map on which they are to move, and a description of their destinations.

\section{DEMONSTRATION}

Our interactive experiment framework has been used on several occasions, including a demonstration entitled "Interactive Mesh Network Planning" at the Interop Tokyo 2014 annual trade fair for information technology, where it received the Special Jury Best of Show Award.

The interactive demonstration that we shall perform takes place as follows (please refer to Figure 4 for an overview):

- Video traffic from the camera of the tablet PC located at the demonstration booth is sent to an emulated node which is assigned to a virtual location on the left-hand side of the screen;

- Participants have the task to use the available network resources in order to create a multi-hop mesh network topology, so that the video traffic can be streamed to another emulated node, which is assigned to a virtual location on the right-hand side of the screen;

- If the network planning is successful, video is shown on a display located at the demonstration booth; a score (based on packet loss information) is indicated in the top-left corner of the user interface.

The following virtual network resources are available for building the mesh network:

- A total of 5 buildings, which are the end nodes and intermediate hops of the mesh network; their location cannot be changed by participants;

- A total of 30 cars forming a vehicular network; cars cannot be directly controlled by the user, but they are assigned random destinations at each run and move according to this information;

- Up to 10 wireless towers, which can be placed by the participants on the virtual map and moved to any location throughout the demonstration;

- Up to 10 unmanned aerial vehicles (UAVs) that can be placed by the participants on the virtual map at any

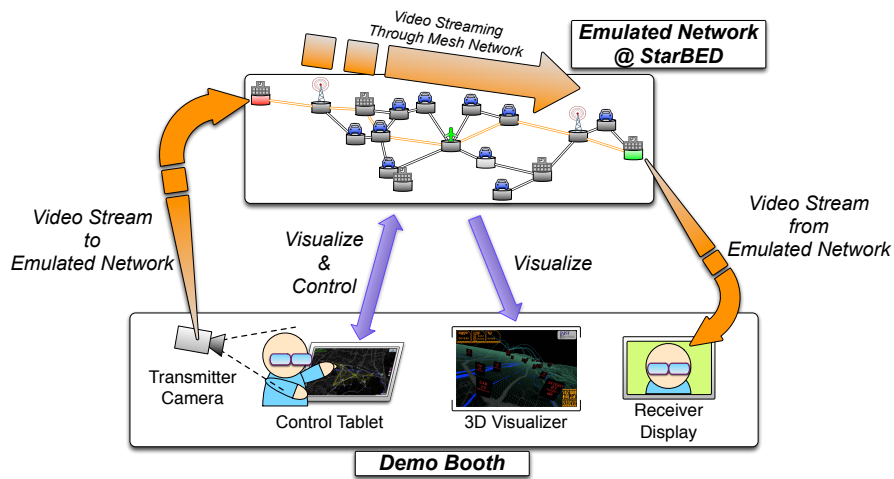

Figure 4: Overview of the demonstration setup.

location throughout the demonstration; the UAVs will then perform a predefined circular motion.

\section{CONCLUSIONS}

We have presented an interactive experiment framework that makes it possible to dynamically change experiment scenarios through direct user input. This framework was used in a demonstration at TridentCom 2015 that asked participants to interactively build a multi-hop mesh network so as to deliver a video stream between two virtual remote location in the experiment area. Live video traffic from the demonstration premises was used as input, and the output was displayed on the demonstration premises as well.

We plan to use the participants' feedback from this and previous demonstrations in order to finalize the design and implementation of the interactive experiment framework, so that it can be made available as a deployable package.

\section{REFERENCES}

[1] R. Beuran, J. Nakata, T. Okada, L. T. Nguyen, Y. Tan, and Y. Shinoda. A Multi-purpose Wireless Network Emulator: QOMET. In Proceedings of the 22nd IEEE International Conference on Advanced Information Networking and Applications (AINA 2008) Workshops, FINA 2008 symposium, pages 223-228, 2008.

[2] R. Beuran, L. T. Nguyen, T. Miyachi, J. Nakata, K. Chinen, Y. Tan, and Y. Shinoda. QOMB: A Wireless Network Emulation Testbed. In Proceedings of the IEEE Global Communications Conference (GLOBECOM 2009), 2009.

[3] M. Carbone and L. Rizzo. Dummynet Revisited. ACM SIGCOMM Computer Communication Review, 40(2):12-20, 2010.

[4] A. Keränen, J. Ott, and T. Kärkkäinen. The ONE Simulator for DTN Protocol Evaluation. In Proceedings of the 2nd International Conference on Simulation Tools and Techniques (SIMUTools '09), 2009.

[5] T. Miyachi, K. Chinen, and Y. Shinoda. StarBED and SpringOS: Large-scale General Purpose Network Testbed and Supporting Software. In Proceedings of the Intl. Conf. on Performance Evaluation Methodologies and Tools (Valuetools 2006), 2006.

[6] OLSR.org. olsrd: an ad hoc wireless mesh routing daemon. http://www.olsr.org/. 